\title{
Comparative Study of Children with/without Iron Deficiency Anemia Based on Parents' Knowledge, Attitude and Practice in Basra/Al-Madinah City
}

\author{
Haydar Mohsin Abdulhussein ${ }^{1}$, Jabbar Taresh Ahmed², \\ Abbas Ali AL-Kinani ${ }^{3}$, Faiz hussien ali $^{4}$, Rasha Mohammed Jumaah ${ }^{5}$ \\ ${ }^{1}$ Postgraduate Student Iraqi at Community Health Techniques, Health and Medical Technical College, Southern \\ Technical University, Iraq, ${ }^{2}$ Assistant Professor, Community Health Techniques, Health and Medical Technical \\ College, Southern Technical University, Iraq, ${ }^{3}$ Specialist in Epidemiology \& Biostatistics, Wasit Health \\ Department, Iraqi Ministry of Health, Iraq, ${ }^{4}$ Specialist in Pediatrics Medicine, Al Madinah General Hospital, \\ Basra Health Department, Iraqi Ministry of Health, Iraq, ${ }^{5}$ Scolar Researcher, Specialist in Communicable \\ Diseases, Wasit Health Department, Iraqi Ministry of Health, Iraq
}

\begin{abstract}
Background: Children under five years of age suffer from anemia is a common medical problem worldwide. Iron deficiency anemia is the most prevalent in infants and has proven to be a public health issue. Many studies have been conducted on anemia among infants but the scope of the studies focused on anemia prevalence with less emphasis on anemia knowledge and parental practice.

Objective: To explore the level of parental knowledge, attitude, and practice towards iron deficiency anemia.

Method: A cross-sectional study in the first taken 150 children that attended to Children's Counseling at Madinah General Hospital from these in the second case control study show only 30 children that effect with IDA and 30 children without IDA.

Results: In this study, the prevalence of anemia in less than two years is $46.7 \%$. knowledge answers near half study sample with their children previously diagnosed with IDA was $16(53 \%)$, the attitude of parents about IDA between two groups not there is a significant relationship and most answers of parents with their children previously diagnosed with IDA was between Fair 17 (57\%) and Poor 11(36\%), not there is a smaller study in this article to refuse or accepted. Practices show a significant association between (case with IDA) and (control without IDA) because (65\% poor) practice About IDA in the case in other side control was (26\%) poor only About IDA in the differential in practice refer more vulnerable to the effect of children to IDA.
\end{abstract}

Conclusion: Anemia is prevalent in infants 24 months old with substantial differences in parents' knowledge and practices.

Keywords: Comparative study, Iron deficiency anemia, Knowledge, Basra.

\section{Introduction}

\section{Corresponding Author:}

Abbas Ali AL-Kinani

Specialist in Epidemiology \& Biostatistics, Wasit Health Department, Iraqi Ministry of Health, Iraq e-mail: abbasalkanani08@gmail.com
Anemia is one of the most widely held audiences Problems with health, in particular in developing countries. Its health, social and economic importance Following. Anemia According to WHO the most common nutritional deficiency due to iron is Worldwide disorder. Two billion people - more than 30 per cent 
The world $>$ s population - anemic, mostly because of iron Deficit ${ }^{1}$

Children under five years of age suffer from anemia is a common medical problem worldwide. Iron deficiency anemia is the most prevalent in infants and has proven to be a public health issue. Many studies have been conducted on anemia among infants but the scope of the studies focused on anemia prevalence with less emphasis on anemia knowledge and parental practice. In Iraq, iron deficiency (ID) prevalence in children under the age of five is $14.4 \%$, and IDA prevalence is $6.8 \% .^{2}$

Aim of the Study: To explore the level of parental knowledge, attitude and practice towards iron deficiency anemia.

\section{Method}

1. Study Settings: In the Basra/Iraq, Children with clinical signs of undernutrition were randomly selected from the Children>s Counseling at Madinah General Hospital by a pediatrician and newborn specialist in the Al-Madinah areas, north of Basra Governorate, and laboratory investigation doing for him including hemoglobin $(\mathrm{Hb})$ and serum ferritin measurement at nine months to 83 months.

2. Study Design: This is case control study.

3. Data Collection: Data was collected over three months, from $7^{\text {st }}$ January to $18^{\text {nd }}$ April, 2020. Data (using a structured questionnaire tool) was obtained from the parents of eligible infants through interview (face to face).

4. Data Collection Tool: The data collection tool was based on medical references and previous researches The content validity was tested via ten pediatric consultants working in Ministry of Health. The questionnaire is composed of three parts: the first part is about the sociodemographic data of the parents. The second part includes questions to explore the knowledge of parents about iron deficiency anemia,

Part Two: Laboratory measurements

This part of the questionnaire consists of questions related to child-related measures such as hemoglobin level, serum ferritin level according Normal findings ${ }^{3}$

Hemoglobin $=1-6$ years: $9.5-14 \mathrm{~g} / \mathrm{dL}$

Ferritin $=$ Newborn: $25-200 \mathrm{ng} / \mathrm{mL}$ $\leq 1$ month: $200-600 \mathrm{ng} / \mathrm{mL}$

2-5 months: $50-200 \mathrm{ng} / \mathrm{mL}$

6 months- 15 years: $7-142 \mathrm{ng} / \mathrm{mL}$

Iron deficiency was defined as serum ferritin levels of below $31 \mathrm{ng} / \mathrm{ml}$, while iron-deficiency anemia was defined as hemoglobin of less than $11 \mathrm{~g} / \mathrm{dl}$ and serum ferritin of less than $31 \mathrm{ng} / \mathrm{ml}^{4}$

5. .Exclusion criteria: Children with hereditary blood diseases: thalassemia, sickle cell anemia and macular (G6PD) glucose 6 phosphate dehydrogenase deficiency excluded from this study.

\section{Measures and Parameters of KAP study}

Regarding the Knowledge: The scale of the three levels was rated on the 3 points (likert respondent scale) it was scored as a scoring of agreed about by assigning a score of (3) for yes, (1) for (no) and score of (2) for I don't know.

Number of questions: 27

$$
\text { Minimum }=27, \text { Maximum }=81, \text { Medium }=64
$$

The medium was calculated for each participant and those score below the medium (score $<64$ ) consider poor score, (score $>64)$ good and acceptable score.

Questions was about Do you know what iron deficiency, symptoms of iron deficiency, causes of iron deficiency, iron rich food, the negative effects of iron deficiency, the source of your information about iron deficiency anemia, Tea, coffee and milk are among the substances that reduce the body's proper absorption of iron, A balanced diet should contain the following nutrients, Iron deficiency anemia is one of the most common types and dietary anemia types.

Regarding Attitude: A scoring of attitude divided into two score agreed about by assigning a score of (2) for the answer by (agree), (1) for the answer by (disagree).

Number of question: 10

Minimum $=10$, Maximum $=20$, Medium $=15$

The medium was calculated for each participant and those score below the medium (score $<15$ ) consider poor score, (score $>15$ ) good and acceptable score.

Attitude was about are you with a child drinking tea with a meal, the child to drink iron drink after eating, 
iron drink children before eating, the child eating fruits regularly, eating red meat, eating ready-made juices, drinking cola and soft drinks, eating crackers (chips and derivatives), eating breakfast daily, eating lunches daily and eating dinner every day.

Regarding Practices: A scoring of practices agreed about by assigning a score of (2) for the answer by (yes) and (1) for the answer by (no).

Number of question: 13

Minimum $=13$, Maximum $=26$, Medium $=24$

The medium was calculated for each participant and those score below the medium (score $<24$ ) consider poor score, $($ score $>24)$ good and acceptable score.

Practice question was about does the child have tea, drink tea with food or immediately after eating, eat vegetables (spinach, parsley, radish, basil), eat fruits regularly, eat red meat, eat chicken, eat eggs, eat readymade juices, drink cola and soft drinks weekly, eat crackers (chips and derivatives), eat breakfast daily, eat lunch daily and eat dinner every day

Statistical analysis: Descriptive, frequencies, central tendency, ANOVA, chi-square, correlation have been used to clarify the relationship between the research variables. The Statistical Package for the Social Sciences (SPSS) version 24 was used in the statistical analysis and treatments.

The significance of different percentages (qualitative data). Statistical significance was considered whenever the $\mathrm{P}$ value was equal or less than 0.05 .

\section{Results}

Data are collected orally by an interviewer using a standardized questionnaire. The age group of children of the study was 46.7 between (9-23) month was suffering from IDA, and 43.3 control group, 72-83 month with high significant association between age group and study sample. This differential may be because of a split of age group to categories.

In another variable from DGDVs show not has the association between group (case \& control) but only was weak a significant association in variable crowding index P.Value $<0.05$ half of the crowding index case was (2.1 - 4) and in control was (4.1->5) person per bed in the room.

KAP study shown in figure (1) compressed between parents of children that attend to children's constitution asked about KAP about IDA.

Show in figures of knowledge does not have a significant association because the answer to the question nearing.

Also in figure attitude show non-significant association $P$. Value $>0.05$ because their attitude about IDA was smaller PV (0.024), but figure practice show a significant association between (case with IDA) and (control without IDA) because (65\% poor) practice About IDA in the case in other side control was $26 \%$ poor only About IDA in the differential in practice refer more vulnerable to the effect of children to IDA.

In table (1) show correlations between DGVs and KAP study show a positive association between practices age group, occupation of father, and knowledge $(\mathrm{P}$. Value $<0.05)$ respectively, also show a significant association between knowledge and SES (P.Value < $0.05)$ and gender (P.Value $<0.05)$. 


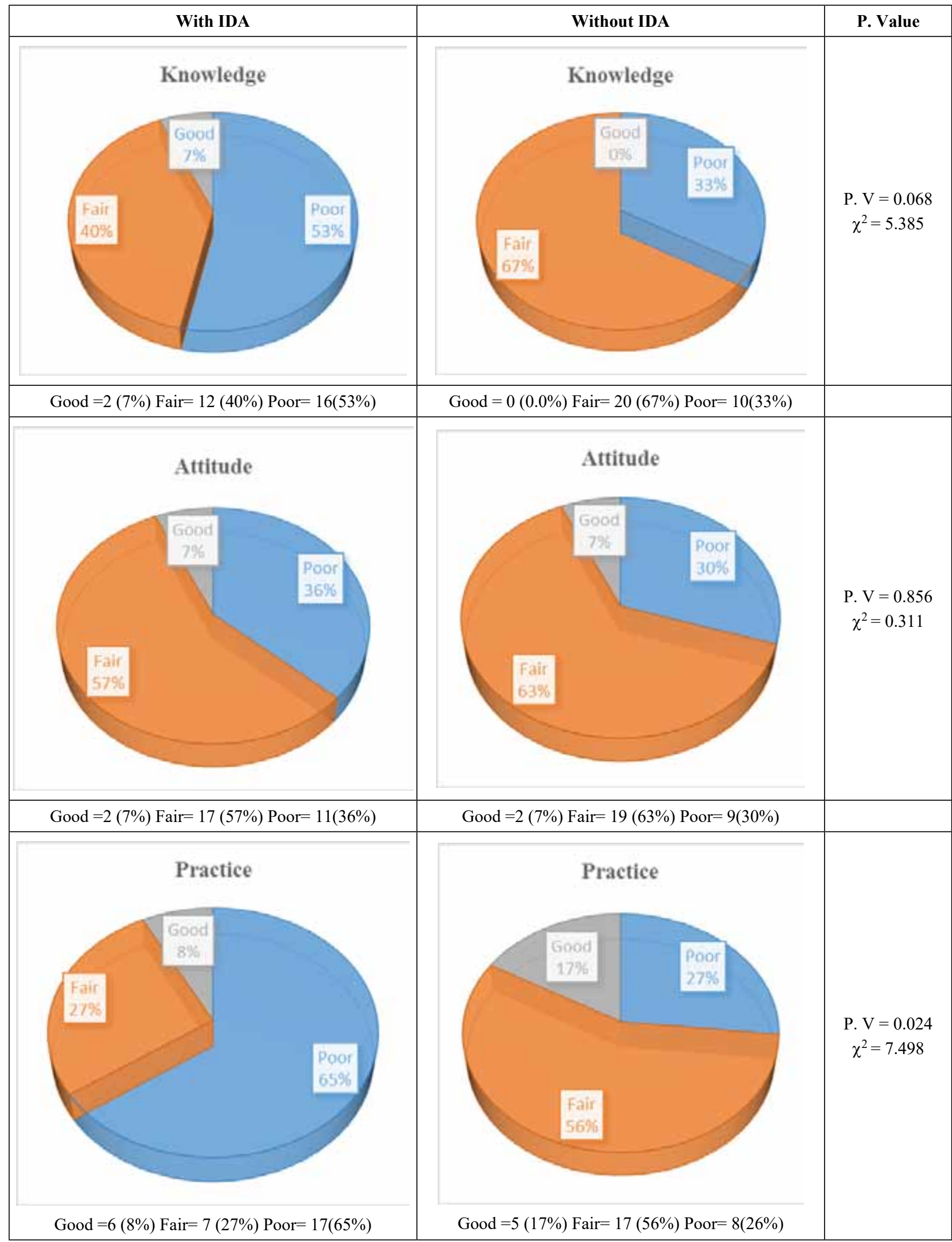

Figure (1) 
Table (1): Correlations between Demographic and socioeconomic characteristics with KAP study

\begin{tabular}{|c|c|c|c|c|c|c|c|c|c|c|c|c|c|}
\hline Corr & elations & & $\hat{z}$ & $=$ & $\ddot{\mathscr{E}}$ & 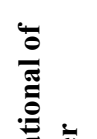 & 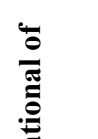 & 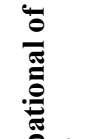 & 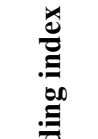 & & 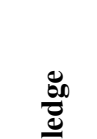 & $\approx$ & \\
\hline & $\mathrm{s}-\mathrm{g}$ & C.C & .202 & & & & & & & & & & \\
\hline & Dex & Sig. & .061 & & & & & & & & & & \\
\hline & Daridona & C.C & .018 & .114 & & & & & & & & & \\
\hline & Testatiot & Sig. & .445 & .193 & & & & & & & & & \\
\hline & Educational & C.C & .034 & $-.120-$ & .101 & & & & & & & & \\
\hline & of mother & Sig. & .397 & .180 & .221 & & & & & & & & \\
\hline & Educational & C.C & $-.219-^{*}$ & $-.278-^{*}$ & $\begin{array}{l}.083- \\
-\end{array}$ & $.304^{* *}$ & & & & & & & \\
\hline & of father & Sig. & .047 & .016 & .265 & .009 & & & & & & & \\
\hline & Occupational & C.C & $-.060-$ & -.099 & $-.035-$ & $-.121-$ & .104 & & & & & & \\
\hline$\&$ & of father & Sig. & .323 & .226 & .397 & .178 & .214 & & & & & & \\
\hline $\begin{array}{l}\bar{B} \\
n \\
n\end{array}$ & Crowding & C.C & .138 & $.521^{* *}$ & .171 & $-.049-$ & $-.266-^{*}$ & .029 & & & & & \\
\hline 胥 & index & Sig. & .146 & .000 & .096 & .355 & .020 & .412 & & & & & \\
\hline$\frac{\pi}{\tilde{D}}$ & S.ES & C.C & $-.129-$ & $-\underset{* *}{-371-}$ & $-.202-$ & $.493^{* *}$ & $.707^{* *}$ & $-.189-$ & $-\underset{* *}{-.530-}$ & & & & \\
\hline & & Sig. & .163 & .002 & .061 & .000 & .000 & .074 & .000 & & & & \\
\hline & Vnouldo & C.C & .040 & $-.236-^{*}$ & $-.110-$ & $-.113-$ & .170 & .110 & $-.179-$ & $.227^{*}$ & & & \\
\hline & Anowreage & Sig. & .381 & .035 & .202 & .195 & .097 & .201 & .086 & .040 & & & \\
\hline & Attitude & C.C & $-.146-$ & .081 & .194 & .212 & .041 & .010 & .022 & .054 & .071 & & \\
\hline & Alitude & Sig. & .133 & .268 & .068 & .052 & .379 & .470 & .434 & .340 & .295 & & \\
\hline & Drontip & C.C & $.268^{*}$ & $-.140-$ & -.079 & .125 & .169 & $.408^{* *}$ & .086 & .007 & $.307^{* *}$ & .016 & \\
\hline & Practice & Sig. & .019 & .143 & .273 & .171 & .098 & .001 & .258 & .478 & .009 & .453 & \\
\hline & 0 & C.C & $.647^{* *}$ & $.237^{*}$ & .156 & .090 & .098 & $-.168-$ & $.243^{*}$ & .034 & .154 & .062 & .211 \\
\hline & IDA & Sig. & .000 & .034 & .117 & .248 & .228 & .099 & .031 & .399 & .120 & .318 & .050 \\
\hline
\end{tabular}

*Correlation is significant at the 0.05 level (1-tailed). (C.C $=$ Correlation Coefficient)

**Correlation is significant at the 0.05 level (1-tailed).

\section{Discussion}

Our study showed that the prevalence of anemia in infants aged 9 months who took part in this research was $46.7 \%$, which is in concordance with previous statistics and local studies Al-jamri A. ${ }^{5}$

Residence distribution of the sample mostly lives in rural, because the hospital locating in rural and most of attended to hospital from this area. Show the high affected with a decrease in was 28 (93.3) from the total study, this accepted with Saaka M. and Gebreweld A. ${ }^{6,7}$
In your articles, it was found that children with anemia in rural and urban areas had a high rate of (73.0) \& (59.5) respectively.

Occupation of the parent that have children affected with IDA most distribution in side father was unemployed 18 (60.0), in other side occupation of the mother was housewives 60 in total study sample, this agree with Gebreweld A. ${ }^{7}$ In his article it was found that occupation a high percentage among housewives was (43.1). 
Overcrowding has affected many health problems one of thesis nutrition status of children show in our study crowding index most distribution between (2.14) that has children affected with IDA was 15 (50.0), this disagree with Abdel-Rasoul G. ${ }^{8}$ Most distribution is between $\leq 2$ they have IDA 36 (76.6).

This study was mostly on children with lowsocioeconomic status. Low socioeconomic status has been known to be a risk factor of IDA. However, we found that our prevalence of IDA is 18 (60.0) suffering from low scale of S.E.S this study accepted with Andriastuti M. ${ }^{9}$ The overall prevalence of IDA in them study was 14.0 .

Knowledge: Show in our results knowledge answers near half study sample with their children previously diagnose with IDA was 16 (53) this results accepted with Al-jamri A. ${ }^{5} 32.5 \%$ of parents correctly answered half or more of the questions regarding IDA complications.

Attitude: Funding attitude of parents about IDA between two group not there is significant relationship and most answers of parents with their children previously diagnose with IDA was between Fair 17 (57\%) and Poor 11(36\%), not there is smmiller study in this prochect to refuse or accepted.

Practices: Show a significant association between (case with IDA) and (control without IDA) because (65\% poor) practice About IDA in the case in other side control was $26 \%$ poor only About IDA in the differential in practice refer more vulnerable to the effect of children to IDA, this result different with Abdallah R. ${ }^{10}$ demonstrates the mean values of food habits of the anemic cases and the normal control. It can be noticed that there were no statistically significant differences between cases and the normal control in food habits as, the $P$ value $>0.05$.

\section{Conclusion}

This finding from this study suggested that PARENTS of children had less awareness of iron deficiency anemia and significant difference in practice between groups of study.

Practice will play a significant role in enhancing anemia and positive correlation between Knowledge, occupation of father, and age group with practice towards iron deficiency anemia.
Ethical Consideration: The Science Ethical Committee of the Ministries of Environmental and Health and Higher Education and Scientific Study in Iraq, by ethical approval.

Conflict of Interest: The writers claim no conflict of interest.

Funding: Self

\section{Reference}

1. Abdel GM, Elgendy FM, Abd ML. Iron deficiency anemia among preschool children $(2-6$ years $)$ in a slum area (Alexandria, Egypt): an intervention study. 2015:213-220. doi:10.4103/11102098.211534

2. Jaber RZ, Al-salait SK. Microcytosis in children and adolescents with the sickle cell trait in. 2019;54(1).

3. Pagana KDPTJPTN. MOSBY'S DIAGNOSTIC AND LABORATORY TEST REFERENCE, FOURTEENTH EDITION. (Pagana KDPTJPTN, ed.). St. Louis, Missouri 63043: Elsevier

4. AishatOluwatoyin Sakaa, Ayodele I. Ojuawoa, Mohammed Jimoh Sakab, S. Abayomi Biliaminuc LO. Assessment of iron deficiency in malnutrition: the value of serum ferritin. Tanta Med J. 2019:239244. doi:10.4103/tmj.tmj

5. Al-jamri A, Al-awainati F, Ali M, Jaafar M, Hussain M, Jassim G. Parents ' Knowledge and Practice of Iron Therapy for Anemic Infants in Primary Health Care in Bahrain. 2017;9(8). doi:10.5539/gjhs. v9n8p99

6. Saaka M, Galaa SZ. How is dietary diversity related to haematological status of preschool children in Ghana ? Food Nutr Res. 2017;61(1). doi:10.1080/1 6546628.2017.1333389

7. Gebreweld A, Ali N, Ali R, Fisha T. Prevalence of anemia and its associated factors among children under five years of age attending at Guguftu health center, South Wollo, Northeast Ethiopia. PLoS One. 2019;14(7):1-13. doi:10.1371/journal. pone. 0218961

8. Abdel-Rasoul G, Elgendy F, Abd Elrazek M. Iron deficiency anemia among preschool children (2-6 years) in a slum area (Alexandria, Egypt): an intervention study. Menoufia Med J. 2017;30(1):213. doi:10.4103/1110-2098.211534 
9. Andriastuti M, Ilmana G, Nawangwulan SA, Kosasih KA. Prevalence of anemia and iron profile among children and adolescent with low socio-economic status. Int J Pediatr Adolesc Med. 2019;(xxxx). doi:10.1016/j.ijpam.2019.11.001
10. Ya, Ghi AR and MS, bn Gaza A-AU-, et al. Iron Deficiency Anemia Among Kindergarten Children Living in Marginalized Areas in Gaza Strip Submitted by/Abdallah R . Yaghi. thesis. 2013. 